\title{
Self-assembly of organic-inorganic hybrid nanolayers: effect of endgroup polarity on nanostructures
}

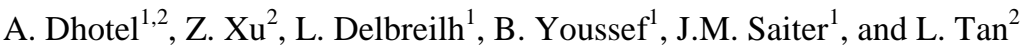 \\ ${ }^{1}$ AMME-LECAP, EA4528, Institut des Matériaux de Rouen, Université et INSA de Rouen, B.P 12, 76801 Saint \\ Etienne du Rouvray Cedex, France \\ ${ }^{2}$ AMME-A-TEAM, Department of Mechanical and Materials Engineering, University of Nebraska, Lincoln, Nebraska \\ 68588, USA
}

\section{Introduction}

Molecular self-assembly (MSA) offers a convenient way to elaborate highly ordered and lightweight nanostructures with molecular-level control of material structure, composition, and dimensions. This sub-branch of supramolecular chemistry which focuses on the spontaneous and reversible construction of ordered aggregates through weak intermolecular interactions represents a facile route to produce organic-inorganic hybrid nanolayers with long-range ordering.

Over the past twenty years, many efforts have been devoted to the construction and structural optimization of well-ordered organic-inorganic nanolayers from monofunctional organosilane surfactants [1-3]. The organization process of such building blocks has been extensively investigated and is known to be governed mainly by the hydrophobic effect arising from a competition of attractive and repulsive interactions between inorganic polar heads, organic nonpolar tails, solvent and water molecules [4, 5]. Such interactions guide the nonpolar chains to aggregate and polar heads to maximize their exposure to the surrounding aqueous medium thus forming structures such as bilayers [6], micelles [7], or vesicles [8]. Finally, van der Waals forces appear between alkyl chains whereas hydrogen bonds and then stronger covalent bonds are formed between inorganic heads thus conferring thermal and mechanical stability to films $[9,10]$.

This study aims to investigate the effect on nanolayer structures of a second polar group located at the chain termination of alkylsilane building blocks. As intermolecular interactions which direct the assembly process are strongly dependent on the chemical composition of precursors, it is expected that the presence of a second polar endgroup will significantly affect these weak interactions thus leading to an alternative structure of nanolayers.

Monofunctional hexadecyltrimethoxysilane (HDTMS) and bifunctional 11bromoundecyltrimethoxysilane (BUDTMS) were used independently as building blocks to produce organicinorganic nanolayers. Structures of these solid thin films were then sequentially characterized and compared after solution assembly and deposition on silicon substrates.

\section{Experimental}

\subsection{Materials}

Hexadecyltrimethoxysilane (technical, $\geq 85 \%$ ) was purchased from Fluka. 11-bromoundecyltrimethoxysilane $(\geq 95 \%$ ) was purchased from Gelest. Hydrogen peroxide, and sulfuric acid were purchased from Sigma-Aldrich. Tetrahydrofuran (THF) was purchased from VWR International and Fisher Scientific. Hydrochloric acid was purchased from Merck. All these chemicals were used as received without further purification. The $500 \mu \mathrm{m}$ prime grade silicon wafers, $\mathrm{P}(100) 10-20 \mathrm{ohm}-\mathrm{cm}$ and $\mathrm{P}(100) 1$ $10 \mathrm{ohm}-\mathrm{cm}$, were purchased from University Wafer and Siltronix. After being cut into rectangular pieces $(1.0 \mathrm{x}$ $2.0 \mathrm{~cm}^{2}$ ), they were rinsed with water, and sequentially sonicated for $15 \mathrm{~min}$ in ethanol and acetone. Their surfaces were then hydroxylated in a piranha solution (70:30, $\mathrm{H}_{2} \mathrm{O}_{2}: \mathrm{H}_{2} \mathrm{SO}_{4}$ ) for $30 \mathrm{~min}$, copiously rinsed with deionized (DI) water and dried under a stream of gaseous nitrogen.

\subsection{Nanolayer Preparation}

Alkylsilane nanolayers were prepared in solution by adding precursors into a mixture of THF, DI water, and hydrochloric acid according to the following molar ratios HDTMS:THF: $\mathrm{H}_{2} \mathrm{O}: \mathrm{HCl}=0.85: 100: 7: 0.002$ and BUDTMS:THF: $\mathrm{H}_{2} \mathrm{O}: \mathrm{HCl}=0.95: 100: 9: 0.006$. The mixture containing HDTMS was then stirred at $400 \mathrm{rpm}$ for $3 \mathrm{~h}$ at room temperature while the BUDTMS containing solution was stirred at $400 \mathrm{rpm}$ for $6 \mathrm{~h}$ at $5^{\circ} \mathrm{C}$. Solutions were then dropcasted or spincoated (3000 rpm) onto hydroxylated silicon wafers. Films were then dried inside a covered Petri dish for $24 \mathrm{~h}$ at room temperature. 


\subsection{Structural Characterization}

X-ray scattering patterns were obtained either on a Bruker-AXS D8 Discover diffractometer or a Rigaku D/Max-B Geigerflex diffractometer. Both diffractometers use a $\mathrm{Cu} \mathrm{Ka}(1.544 \AA)$ radiation. Digital data were recorded from $2 \theta=1^{\circ}-16^{\circ}$ at an angular resolution of $0.02^{\circ}$ and angular velocity of $0.4^{\circ} \cdot \mathrm{min}^{-1}$. Thin films were analyzed directly on their silicon substrates. A Dimension 3100 SPM atomic force microscope was used at room temperature to obtain topographic images of thin films. Images were recorded at a maximum resolution of 512 lines under a tapping mode. Molecular dynamics (MD) simulations were performed assuming a constant-pressure and constant-temperature (NPT) ensemble. The temperature is controlled at $300 \mathrm{~K}$, and the external pressure is set at $1 \mathrm{~atm}$. The consistent valence force field (CVFF) is selected to describe interactions among molecules.

\section{Results}

in our experimental protocol, molar ratios among components, solvent type, and precursor natures were chosen to form multilayer films with a lamellar structure where each lamella (referred to as nanolayers thereafter) is composed of tightly packed molecules oriented along the substrate normal $[1,9]$.

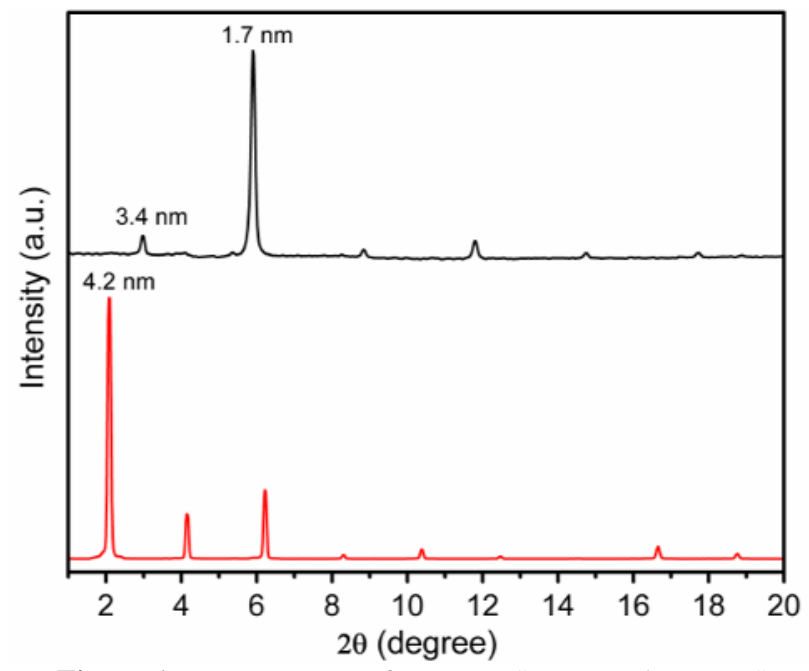

Figure 1. XRD patterns of BUDTMS (top) and HDTMS (bottom) nanolayers.

X-ray diffraction (XRD) was preliminary employed to identify the nanostructures of films. Figure 1 shows XRD patterns recorded on thin films of HDTMS (bottom pattern) and BUDTMS (top pattern). Considering a length of $2.3 \mathrm{~nm}$ for a HDTMS molecule [9], the peak at $2 \theta=2^{\circ}(\mathrm{d}$-spacing $=4.2 \mathrm{~nm})$ suggests that HDTMS nanolayers are composed of bilayers where molecules are slightly tilted and packed into a head-to-head arrangement. Peaks at higher $2 \theta$ values (Figure 1, bottom pattern) correspond to secondary diffraction peaks of bilayers indicating that the lamellar structure of HDTMS films is solely composed of bilayers. In contrast, BUDTMS nanolayers (Figure 1, top pattern) appear to be composed of two distinct molecular organizations. While the weak intensity peak at $2 \theta=3^{\circ}(\mathrm{d}$-spacing $=3.4 \mathrm{~nm})$ can be attributed to the presence of bilayers, assuming a length of $1.8 \mathrm{~nm}$ for a BUDTMS molecule, the high intensity peak at $2 \theta=5.9^{\circ}(\mathrm{d}$-spacing $=1.7 \mathrm{~nm})$ may originate from a second molecular organization. Indeed, the weak dipole moment created by the bromine atom at the termination of the hydrocarbonated chain of BUDTMS (Figure 2a) is expected to affect the assembly process of building blocks and, therefore, the final organization of molecules in films.

(a)

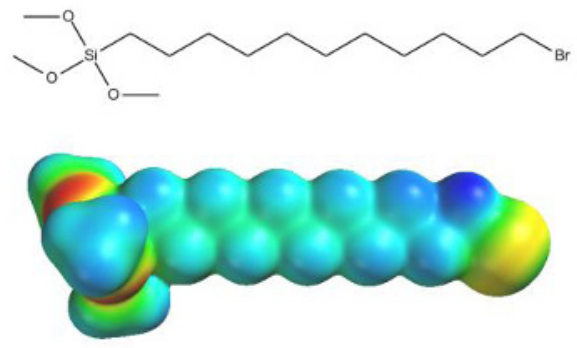

(b)

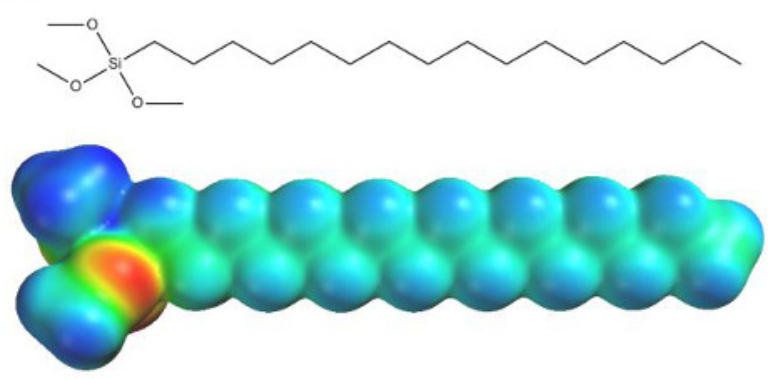

Figure 2. Chemical formula and electrostatic potential surface of (a) BUDTMS and (b) HDTMS molecules.

A d-spacing of $1.7 \mathrm{~nm}$ being very close to the length of a BUDTMS molecule, the peak at $2 \theta=5.9^{\circ}$ may correspond to a monolayer-on-monolayer packing where molecules are stacked on top of each other and oriented in the same direction. An interdigitated organization of molecules also fits a d-spacing value of $1.7 \mathrm{~nm}$. In this lamellar packing mode, alkyl chains of adjacent molecules are alternately pointing up and down [3,9]. Finally, this high intensity peak may also originate from BUDTMS bilayers in which bromine atoms strongly diffract X-rays.

Molecular dynamic (MD) simulations were then conducted to determine which molecular packing is the most thermodynamically stable. As depicted in Table 1, examination of these molecular organizations reveals that the bilayered packing mode exhibits the lowest binding energy value suggesting this configuration as the most energetically favorable. 
Table 1. Calculated average binding energies and layer thicknesses of BUDTMS packing modes examined by MD simulations.

\begin{tabular}{ccc}
\hline Packing Mode & $\begin{array}{c}\text { Binding energy } \\
(\mathrm{kcal} / \mathrm{mol})\end{array}$ & $\begin{array}{c}\text { Layer thickness } \\
(\mathrm{nm})\end{array}$ \\
\hline $\begin{array}{c}\text { Monolayer-on- } \\
\text { monolayer }\end{array}$ & -31.8 & 1.63 \\
$\begin{array}{c}\text { Interdigitated } \\
\text { Layer }\end{array}$ & -28.2 & 2.03 \\
Bilayer & -34.9 & 3.22 \\
\hline
\end{tabular}

The bilayered conformation of BUDTMS molecules in self-assembled films was further confirmed by analyzing the film topography by atomic force microscopy (AFM). As shown in Figure 3, self-assembled BUDTMS films are composed of a stacking of nanolayers (top image). Examining the height profile of these layers (bottom profile) confirm that the height of each nanolayer is $\sim 3.7$ $\mathrm{nm}$ which is in good agreement with XRD and MD simulation results that suggest a bilayered structure of layers.
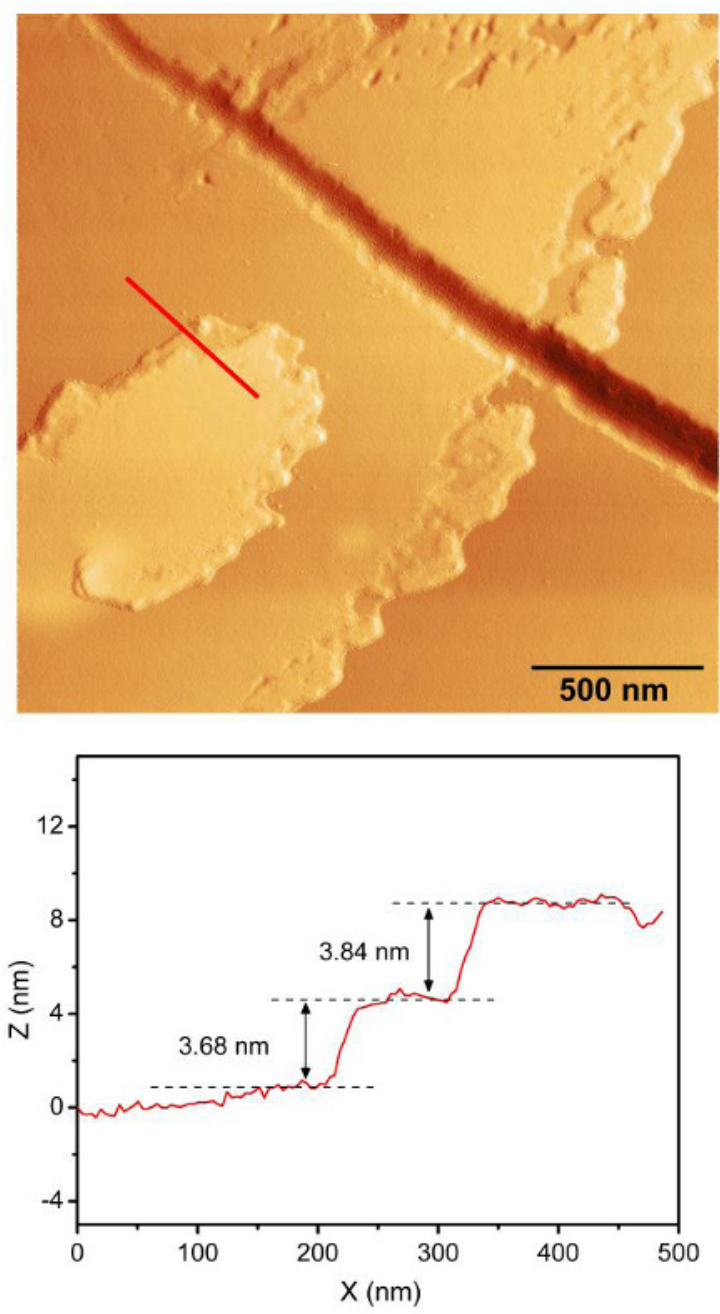

Figure 3. (Top) AFM image highlighting the lamellar structure of self-assembled BUDTMS films. (Bottom) Height profile recorded along the red line on the AFM image confirming the bilayered structure of BUDTMS nanolayers.

\section{Discussion}

Experimental and simulation results suggest that our experimental protocol leads both HDTMS and BUDTMS molecules to self-assemble into a bilayered conformation even though their XRD patterns differ. Likely, this dissimilarity is due to the chemical composition of building block endgroups. Indeed, the limited number of electrons in the methyl termination of HDTMS may prevent endgroups to diffract X-rays making them invisible to XRD. In contrast, within a BUDTMS bilayer, bromine atoms form a high electron density plane in the middle of each nanolayer. The elevated number of electrons within the plane makes it visible to XRD by diffracting X-rays. As a result, BUDTMS bilayers contain two diffraction planes (i.e. the inorganic siloxane network and the plane constituted by the bromine terminations of building blocks) whereas HDTMS bilayers contain only one diffraction plane (i.e. the inorganic siloxane network). Therefore, BUDTMS bilayers are characterized by two set of diffraction peaks (d-spacing $=3.4$ and 1.7 $\mathrm{nm}$ ), while HDTMS bilayers are characterized by a unique set of diffraction peaks $(\mathrm{d}$-spacing $=4.2 \mathrm{~nm}$ ).

\section{Conclusion}

The effect of endgroup polarity on the nanolayer structure of self-assembled alkylsilane thin films was investigated by experimental and simulation techniques. results indicate that the weak dipole moment induced by the bromine atom at the endgroup of budtms has no remarkable effect on the self-assembly process and final nanostructure of films. indeed, both methyl-terminated hdtms and bromine-terminated budtms were found to form a lamellar structure where each lamella is composed of molecules arranged in a bilayered conformation.

Although the formation of bilayered nanolayers from monofunctional alkylsilane molecules has been extensively studied already, the ability to form such structures from bifunctional precursors is much less known. therefore, it is expected that this finding could open up a new range of potential applications of alkylsilane nanolayers by enlarging the number of precursor candidates to more complex bifunctional molecules.

\section{References}

1. A. Shimojima, Y. Sugahara, K. Kuroda, Bull. Chem. Soc. Jpn. 702847 (1997)

2. A.N. Parikh, M.A. Schivley, E. Koo, K. Seshadri, D. Aurentz, K. Mueller, D.L. Allara, J. Am. Chem. Soc. 1193135 (1997)

3. A.B. Bourlinos, S.R. Chowdhury, D.D. Jiang, Y.-U. An, Q. Zhang, L.A. Archer, E.P. Giannelis, Small. 1 80 (2005)

4. Y. Fujimoto, A. Shimojima, K. Kuroda, Chem. Mater. 154768 (2003)

5. A. Dhotel, Z. Chen, L. Delbreilh, B. Youssef, J.-M. Saiter, L. Tan, Int. J. Mol. Sci. 142303 (2013) 
6. A. Dhotel, L. Delbreilh, B. Youssef, J. Jiang, G. Coquerel, J.-M. Saiter, L. Tan, J. Therm. Anal. Calorim. 1 (2012)

7. M.M. Kozlov, E. Opatowski, D. Lichtenberg, J. Therm. Anal. Calorim. 51173 (1998)

8. B.J. Ravoo, Supramolecular Chemistry: From Molecules to Nanomaterials John Wiley \& Sons Ltd, 2012

9. A. Dhotel, H. Li, L. Fernandez-Ballester, L. Delbreilh, B. Youssef, X.C. Zeng, L. Tan, J. Phys. Chem. C. 11510351 (2011)

10. C. Yu, Z. Chen, H. Li, J. Turner, X.C. Zeng, Z. Jin, J. Jiang, B. Youssef, L. Tan, Adv. Mater. 224457 (2010) 